\title{
Augmented Fuzzing with Promotion on Numerical Dependence
}

\author{
Sisi $\mathrm{Li}^{1}$, Jiaxi Ye ${ }^{1+}$, Bin Zhang ${ }^{1}$, and Chaojing Tang ${ }^{1}$ \\ ${ }^{1}$ College of Electronic Science, National University of Defense Technology, China
}

\begin{abstract}
Coverage-guided fuzzing is an effective technique to find software bugs, it is skilled at eliminating the execution on the repeated paths, thus the fuzzing performance is improved. However, its code coverage-centric design is still insufficient in bug detection. On the one hand, the coverage-based schedule tries its best to reduce the execution on the explored paths even if there are some undetected bugs inside. On the other hand, it mutates the input randomly, which makes it inefficient to generate target testcases. As a result, it may miss some subtle bugs despite it has already explored the vulnerable codes. In this paper, we present a prototype, named ADA, to augment bug detection on the executed paths via a promotion on the numerical dependence of triggering the bug. The numerical dependence means some special numerical conditions that need satisfying to trigger the bug. In a nutshell, our tool allows an intended number of executions on the explored paths, and utilizes a novel approximation search algorithm, to produce the testcases that can satisfy the numerical dependence of triggering the bug and crash the program. Moreover, we leverage a critical field identification method to adjust the mutation, which can help the engine to quickly produce the testcases satisfying the numerical dependence. We implement ADA based on AFL and radare2, and evaluate its performance on some benchmarks. The results demonstrate that ADA outperforms some other state-of-the-art coverage-guided fuzzers in discovering the bugs on the executed paths. It is the proof that our approach is effective. Besides, the experimental results also indicate that the introduced overhead by our approach is within an acceptable scope.
\end{abstract}

Keywords: software security, fuzzing, bug detection, numerical dependence, promotion.

\section{Introduction}

Fuzzing [1] has become a critical component in testing the quality of software systems. It discovers program defects by feeding the program with numbers of malformed test inputs and monitoring the unintended program behaviors during the execution. Particularly, coverage-based fuzzing has been widely used by mainstream software companies such as Google, Microsoft and Adobe, due to its advantageous performance. For example, Google’s OSS-Fuzz [2] platform adopts several state-of-the-art fuzzers, including AFL [3], libFuzzer [4] and honggfuzz [5], to continuously test open source applications. Coverage-based fuzzing such as AFL employs lightweight code instrumentation and evolutionary algorithms to retain inputs that discover a new path for further tests, which dramatically improves the efficiency of fuzzing.

Coverage-based fuzzing aims to generate inputs that can achieve maximum code coverage and it is skilled at eliminating the execution on the repeated paths. Once reaching a chunk of code, it will immediately turn to explore other new code regions. Lack of ample tests on the explored paths, it may miss some subtle bugs inside, which is a common challenge for classic coverage-based fuzzing. Besides, it randomly mutates the input to generate new testcases, which makes it hard to search for target testcases. We observe that, to discover certain types of bugs on the explored paths, two conditions need to be satisfied simultaneously: (i) a testcase can execute the vulnerable codes; (ii) the testcase's execution context can satisfy the conditions of triggering the bug. Obviously, it is insufficient for coverage-based fuzzing to discover these types of bugs by merely improving the code coverage of the program.

\footnotetext{
Corresponding author. Tel.: + 86-15116167213;

E-mail address: xiaosatianyu@126.com.
} 
Most of fuzzing techniques are aimed at satisfying the condition $i$, neglecting the condition ii instead. To address the challenge related to the condition ii, different approaches are proposed which typically make use of advanced program analysis techniques, such as taint tracking [6] and symbolic execution [7]. Specifically, taint-based directed fuzzers leverage classical taint analysis to identify the critical input bytes in a seed and fuzz them to achieve a specific value that is required to uncover a bug at a given program location. Besides, the hybrid testing tool SAVIOR [8] verifies the bug along the program path by synthesizing the faulty constraint of triggering the bug and solving the constraint to construct a test input as the proof. However, these approaches associated with taint tracking and symbolic execution usually incur lots of performance overheads and they may weaken the scalability of fuzzing.

To address the challenges of existing fuzzing techniques, we implement a prototype system called ADA, which integrates a novel promotion module into existing coverage-guided fuzzing. Unlike existing fuzzers, ADA can monitor whether the program executes the critical codes, which include the functions related to comparisons and the vulnerable codes, and it launches the promotion module if the critical codes are explored. Note that our promotion module has two advantages: (1) improving the code coverage of the program by producing the testcases that can meet the numerical dependence of breaking through the comparisons in critical functions; (2) augmenting bug detection on the executed paths by repeatedly testing the explored vulnerable codes and generating the testcases that can satisfy the numerical dependence of triggering the bug. The numerical dependence means some special numerical conditions that need satisfying to trigger the bug or break through the comparison. In this paper, we mainly illustrate the second advantage of ADA mentioned above.

In the promotion module, we propose a critical field identification method to assist the searching process, which can help reduce the searching space of the input. Moreover, we design a novel approximation search algorithm, by introducing new guidance information, it can efficiently produce the testcases that satisfy the numerical dependence of triggering the bug and crash the program. We evaluate the performance of ADA using some benchmarks. The experimental results demonstrate that ADA outperforms some other state-ofthe-art coverage-guided fuzzers in discovering the bugs on the executed paths. It is the proof that our approach is effective.

\section{Motivation}

Coverage-based fuzzing schemes are widely used for software testing and bug detection. As is known, a coverage-based fuzzer aims at maximizing the code coverage to traverse paths that may contain bugs. However, its code coverage-centric design only attempts to satisfy the condition $i$, ignoring the condition $i i$ instead. As a result, it may miss some subtle bugs despite it has already explored the vulnerable code paths, which is a common challenge for coverage-based fuzzing. Three specific factors leading to the above challenge can be listed as follow. Firstly, it is unaware of data-flow information, and it just records the information associated with the execution trace to decide whether a new path is traversed, while ignoring the values of variables along the traversed path. Secondly, it quickly moves on after traversing a new path even if there are some undetected bugs inside. Thirdly, it utilizes random mutations to generate new testcases, making it hard to search for a target testcase.

The code snippet in Fig.1 illustrates the challenge mentioned above. Let us consider the integer overflow bug on Line 7. The program takes the input by read() in Line 4 and calls atoi() to obtain the value of length on Line 5. When if condition on Line 6 is met, the program will read length bytes of contents. In this code, length is a signed variable, but read( ) regards it as an unsigned variable. Therefore, when length is less than 0 , read( ) will turn it into a quite large number and read excessive bytes of contents, which eventually crashes the program. On the contrary, when length is a positive number, no matter how many times the code on Line 7 is executed, the integer overflow bug will never be triggered. 


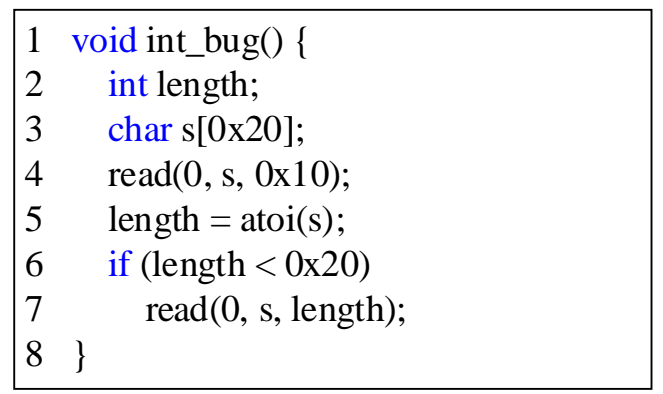

Fig. 1: An example of integer overflow bug.

The classic coverage-based fuzzer AFL may miss the bug on Line 7 even if it has explored the vulnerable code. The reasons for this rather pessimistic behavior are as follows: (1) AFL turns to exploring other new paths when the code on Line 7 is executed and it lacks ample tests on the executed path; (2) AFL mutates the input at random, so it is hard to generate a testcase that can make length a negative.

To address the above limitations of current coverage-based fuzzing, we observe that enough tests on the explored vulnerable code paths and a better searching scheme are demanded. Hence, we propose a novel promotion module based on bug trigger conditions. When the vulnerable code path is detected to have been explored, ample tests on it will be conducted by launching this promotion module, in which an efficient searching scheme is designed to drive the generation of testcases that can trigger the bug. Fig.2 shows the framework of the promotion module, which contains three critical elements denoted in grey:

- The evaluation criterion associated with bug trigger conditions. Code coverage is merely designed to satisfy the condition $i$. Thus, a new evaluation criterion is demanded to guide the searching process, aiming at satisfying the condition ii.

- The evaluation results. The searching scheme gets the evaluation result by calculating the distance between the execution context of the current testcase and that of triggering the bug.

- The modification on testcases. The searching scheme takes the evaluation results as the feedback to modify the testcase, driving the generation of testcases that can trigger the bug and crash the program.

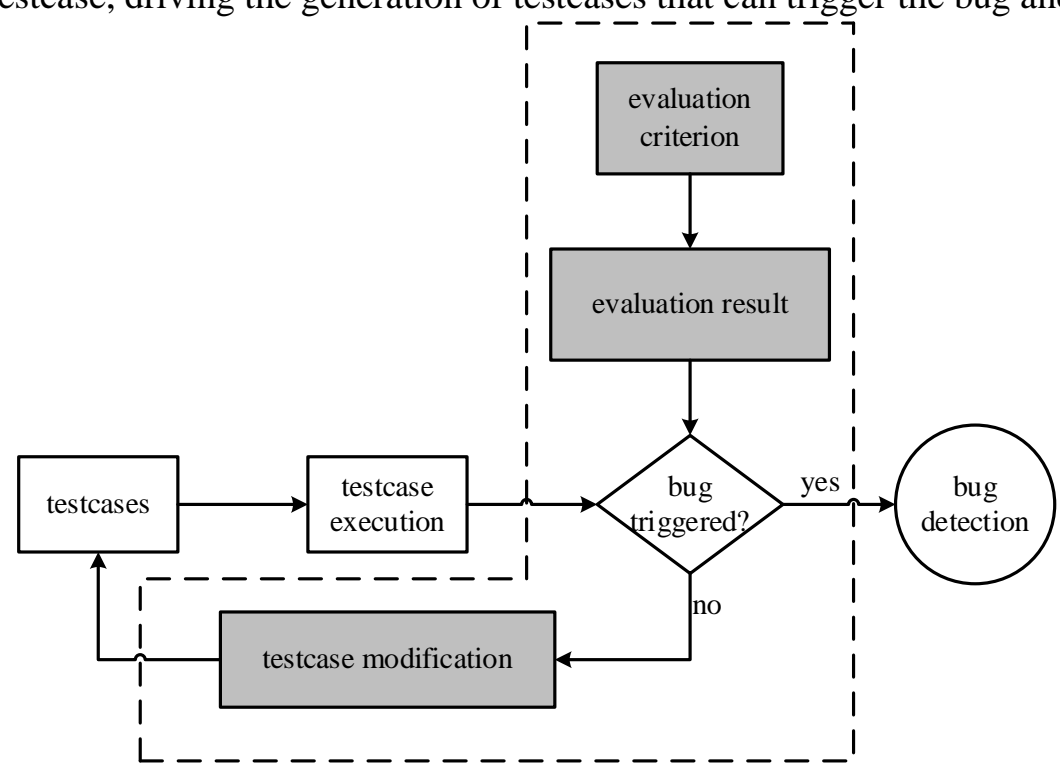

Fig. 2: The framework of the promotion module based on bug trigger conditions.

Note that different types of bugs have different bug trigger mechanisms, and the specific implementation of a promotion module varies with bug trigger mechanisms. In this paper, we implement an augmented fuzzing approach with a promotion on the numerical dependence of triggering the bug, which is aimed to improve the ability of discovering several types of bugs, such as the integer overflow bugs and the format string bugs. The conditions of triggering these types of bugs can be summarized as:

Condition $\boldsymbol{i}$ : a testcase can execute the vulnerable codes;

Condition ii: the testcase's execution context can satisfy the numerical dependence of triggering the bug. 


\section{Design}

\subsection{Numerical Space of Execution Trace}

Each input corresponds to an execution trace. Usually, an input will drive the program to execute a series of instructions, and their execution order constitutes a directed trace. It should be noted that, there are some dynamic variables on the execution trace, and their specific values can be changed in a certain range without deviating from the original execution trace. For example, length in Fig. 1 is a dynamic variable, whose value can be dynamically changed in the range of length $<0 \times 20$. We assume that, for an execution trace $T$, taking the relevant dynamic variables as tensors, the numerical space of the execution trace $T$ will be formed. We denote it as $S_{D}(T)$ and Definition 1 illustrates the concept of $S_{D}(T)$.

Definition 1: For an execution trace $T$, assuming that $v_{1}, . ., v_{m}$ are dynamic variables on $T$, and taking $v_{1}, . ., v_{m}$ as tensors, the numerical space of the execution trace is formed as $S_{D}(T)$. Notably, the values of $v_{1}, . ., v_{m}$ can be only taken from the feasible range of $T$, in which the execution traces won't deviate from $T$.

$$
S_{D}(T)=\left\{\left(v_{1}, \ldots, v_{m}\right) \mid v_{1} \in f_{1}(T), \ldots, v_{m} \in f_{m}(T)\right\}
$$

$v_{m} \in f_{m}(T)$ indicates that the variable $v_{m}$ takes a value from the feasible range $f_{m}(T)$ of the execution trace $T$.

Let us take the codes in Fig.1 as an example to explain $S_{D}(T)$. Assuming that there is an execution trace $T$, whose specific trace is Line $2 \rightarrow 3 \rightarrow 4 \rightarrow 5 \rightarrow 6 \rightarrow 7$. The length is a dynamic variable, and the range of its values on the execution trace $T$ is length $<0 \times 20$. Therefore, the numerical space of $T$ is described as $S_{D}(T)=$ \{length|length $<0 \times 20\}$, and $S_{D}(T)$ can be further divided into two subspaces, one is $S_{D 1}(T)=$ $\{$ length $\mid$ length $<0\}$, and the other is $S_{D 2}(T)=\{$ length $\mid 0 \leq$ length $<0 \times 20\}$. Obviously, the numerical dependence of triggering the integer overflow bug can be satisfied in $S_{D 1}(T)$, but not in $S_{D 2}(T)$. Thus, if only ADA finds out a testcase whose execution context locates in the $S_{D 1}(T)$, the bug can be successfully discovered.

\subsection{Design Details}

The objective of ADA is to augment bug detection on the executed paths via a promotion on the numerical dependence of triggering the bug. The core idea of ADA can be summarized as two points. Firstly, there should be a testcase that can execute the vulnerable codes, that is, condition $i$ is satisfied. Grounded on the condition $i$, we designate the execution trace of the testcase as $T$, and the numerical space of $T$ is denoted as $S_{D}(T)$. Our key insight is that, in order to uncover the bug, the execution context of the testcase must satisfy the numerical dependence of triggering the bug, denoted as $\emptyset$. Secondly, ADA mutates the testcase and promotes the generation of a testcase whose execution context locates in the subspace $S_{D}(T) \cap \emptyset$. Based upon AFL, we design ADA by appending four crucial parts as follow, and the last three parts compose the promotion module.

\subsubsection{Execution Trace Screening}

It is inefficient and needless to apply the promotion approach to all the execution traces. In order to control the overhead, we ought to filter the execution traces and retain those that can pass by the critical codes, which means only the testcases that can execute the critical codes will be used for the promotion process.

This part employs static analysis and dynamic binary instrumentation to screen execution traces. First, based on the prior knowledge [9], ADA uses static analysis to locate the critical codes, which contain the functions related to comparisons and the vulnerable codes. Besides, if a testcase executes the critical codes, dynamic binary instrumentation will be utilized to add a "hit" tag to the testcase, otherwise a "missed" tag will be added. With the tags of "hit" and "missed", ADA is capable of quickly screening out the testcases that explore the critical codes, and merely applies the promotion approach to them, thereby effectively controlling the overhead brought by the searching process.

\subsubsection{Dynamic Variables}

The specific values of dynamic variables are used for two key processes. One is the identification of fields associated with dynamic variables, the other is the heuristic searching process, and these two processes 
will be introduced respectively in the final two parts. Thus, it is imperative to locate the dynamic variables on the execution trace and obtain their specific values.

This part uses static analysis to locate dynamic variables and extract their symbolic expressions. During the test, as soon as the critical codes are executed, ADA will invoke Ptrace [10] to parse the corresponding symbolic expressions, getting the specific values of the dynamic variables. We emphasize that the dynamic variables and the corresponding symbolic expressions are obtained by means of the expert knowledge [9].

Table. 1 shows the dynamic variables of four typical functions and the corresponding symbolic expressions with 64-bit programs. Let us take read() as an example, read() reads a length of contents from the standard input to a specified address, and its third parameter determines the length of contents read from the standard input, for 64-bit programs, this parameter is stored in the $r d x$ register. In hence, the dynamic variable of read( ) is its third parameter, and the corresponding symbolic expression is Read(rdx). When the program executes read( ), we need to parse $\operatorname{Read}(r d x)$ and obtain the value of the $r d x$ register, and we use Ptrace [10] for this purpose. Note that the value of $r d x$ is exactly the value of read( )'s third parameter. Usually, when the Read $(r d x)$ returns a negative, an integer overflow bug will be triggered.

Table. 1: Key codes and symbolic expressions of their dynamic variables with 64-bit programs.

\begin{tabular}{|c|c|c|c|}
\hline Key Codes & Dynamic Variables & Symbolic Expressions & Numerical Dependences \\
\hline memcmp() & two parameters & Read(rdi)、Read(rsi) & memory value comparison \\
\hline strcmp()/strncmp() & two parameters & $\operatorname{Read}($ rdi)、Read(rsi) & string comparison \\
\hline read() & the third parameter & $\operatorname{Read}(\mathrm{rdx})$ & integer overflow \\
\hline printf() & the first parameter & $\operatorname{Read}(\mathrm{rdx})$ & format string \\
\hline
\end{tabular}

In this paper, we describe symbolic expressions of dynamic variables in three ways. First, the value is directly read from a register, such as reading the value of the $r d x$ register, which is denoted as $\operatorname{Read}(r d x)$. The second way is to read the value from a memory, such as reading the value at the address of $0 \times 8048123$, which is denoted as Read (0x8048123). The last way is to read the value from a base address with an offset, and the base address can be a register or a memory, for example, we let $r d x$ be the base address and let $0 x 123$ be the offset, then reading the value at a specified address can be designated as $\operatorname{Read}(\mathrm{rdx}+0 \times 123)$.

\subsubsection{Identification of Key Fields}

As is known, it is quite difficult for fuzzing to search for a target testcase by mutating the entire input offsets. For this reason, we present two types of critical fields, the trace-related fields and the dynamic variable-related fields, and come up with a critical field identification method to reduce the searching space of the input. Our key insight is that, we can effectively reduce the searching space of the input by fixing the trace-related fields and only mutating the dynamic variable-related fields, which makes the searching process less complicated and more efficient. Obviously, in the reduced searching space, the testcases generated have the same execution traces, while the specific values of the dynamic variables on their execution traces are different.

The critical field identification method is designed according to the following two ideas: (1) the execution trace of the testcase varies with the trace-related fields; (2) if the dynamic variable-related fields are changed, the values of the dynamic variables on the execution trace will also change. It should be noted that we only apply the identification method to testcases that can execute the critical codes. The identification process can be illustrated as follow. Firstly, traversing each byte of the testcase (denoted as s1), and mutating each byte separately to generate a new testcase (denoted as $s 2$ ). Secondly, comparing the execution traces of $s 1$ and $s 2$. If their traces are different, the current byte offset will be added to the set of trace-related fields, otherwise we obtain the values of the dynamic variables on their execution traces and further compare them. If the values are dissimilar, the current byte offset will be added to the set of dynamic variable-related fields. Finally, when all the fields of the testcase are traversed, ADA outputs the set of tracerelated fields and that of dynamic variable-related fields. 


\subsubsection{Approximation Search Algorithm}

The random search algorithms in coverage-based fuzzers are inefficient for bug detection. In this part, we put forward a novel approximation search algorithm to produce testcases that can meet the numerical dependence of triggering the bug, thereby augmenting bug detection on the executed paths. Usually, for a numerical dependence $\emptyset$, the relevant dynamic variables have some feasible solutions. Let us consider the integer overflow bug in Fig.1, the numerical dependence of triggering the bug is length $<0$, so length $=1$ is a feasible solution. On the base of the expert knowledge [9], we provide the dynamic variable with a feasible solution, denoted as $V$, so the goal of the search algorithm is to find out a testcase, whose dynamic variable on the execution trace meets $V$. Founded on the feasible solution $V$, the approximation search algorithm is performed on the testcase by fixing the trace-related fields and mutating the dynamic variable-related fields.

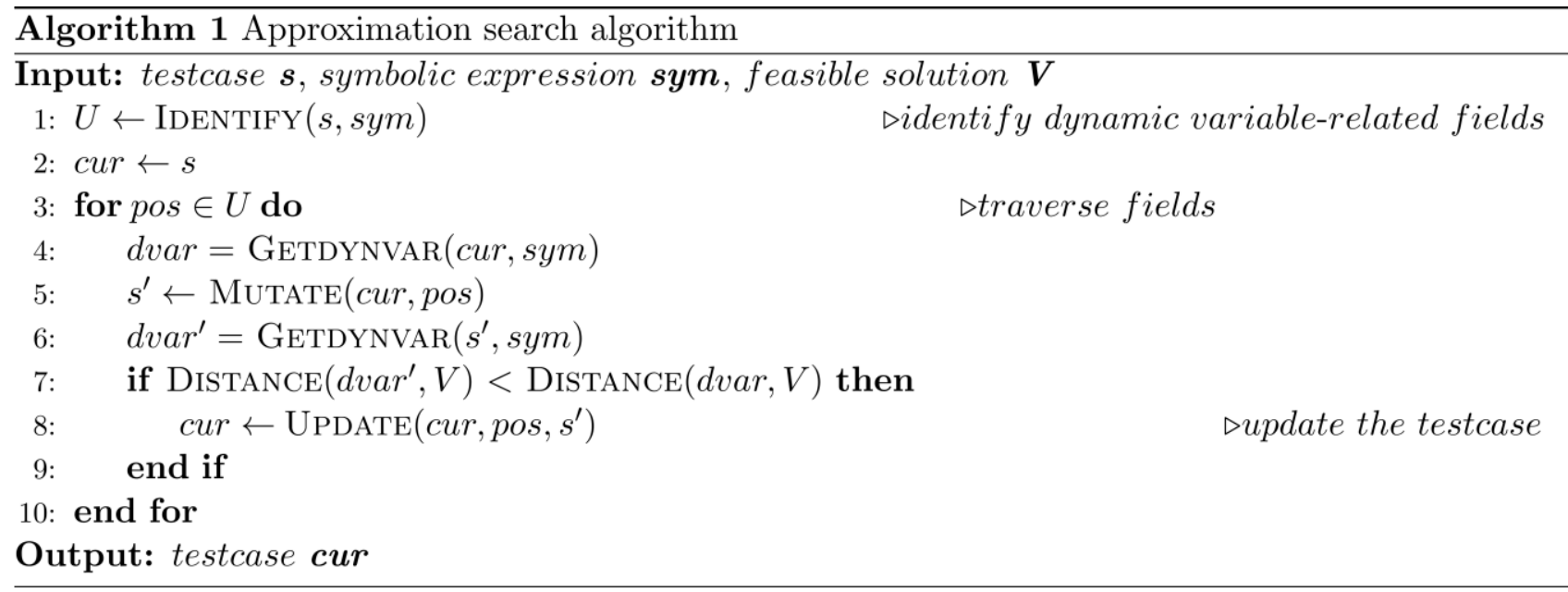

Algorithm 1 shows the heuristic searching process.

As shown in Algorithm 1, for the testcase s, establishing the set $U$, which consists of the dynamic variable-related fields. The algorithm traverses and mutates each field in $U$ to generate a new testcase $s^{\prime}$ on Line 5. Then, it respectively obtains $d v a r$ and $d v a r^{\prime}$, the specific value of the dynamic variable for cur and $s^{\prime}$, and calculates their distances from $V$. If the distance between $d v a r^{\prime}$ and $V$ is smaller, the cur will be updated by replacing the content of cur at pos with the content of $s^{\prime}$ at pos. After traversing all the fields in $U$, the algorithm ultimately outputs the testcase cur.

It is emphasized that the testcase cur generated by Algorithm 1 may not necessarily satisfy the numerical dependence of triggering the bug due to the constraints of the execution context. Nevertheless, ADA will generate numbers of testcases with a "hit" label, by performing the approximation search algorithm on them, ADA can efficiently find out the testcases that satisfy the numerical dependence of triggering the bug.

\subsection{Implementation of ADA}

We have implemented a prototype system ADA. Fig.3 shows the framework of ADA. Specifically, static analysis is implemented using radare2 [11] in Python, which is used to locate the critical codes and obtain the symbolic expressions of the dynamic variables. Dynamic binary instrumentation is implemented using QEMU [12], which aims to screen out the testcases that execute the critical codes by adding a "hit" tag to them. Besides, Ptrace [10] is utilized to obtain the specific values of the dynamic variables by parsing the symbolic expressions, which is implemented in $\mathrm{C}++$. In addition, we extend AFL 2.52b to be the fuzzer in ADA. In a nutshell, ADA is designed to augment bug detection on the executed paths via a promotion on the numerical dependence of triggering the bug or breaking through the comparison. 


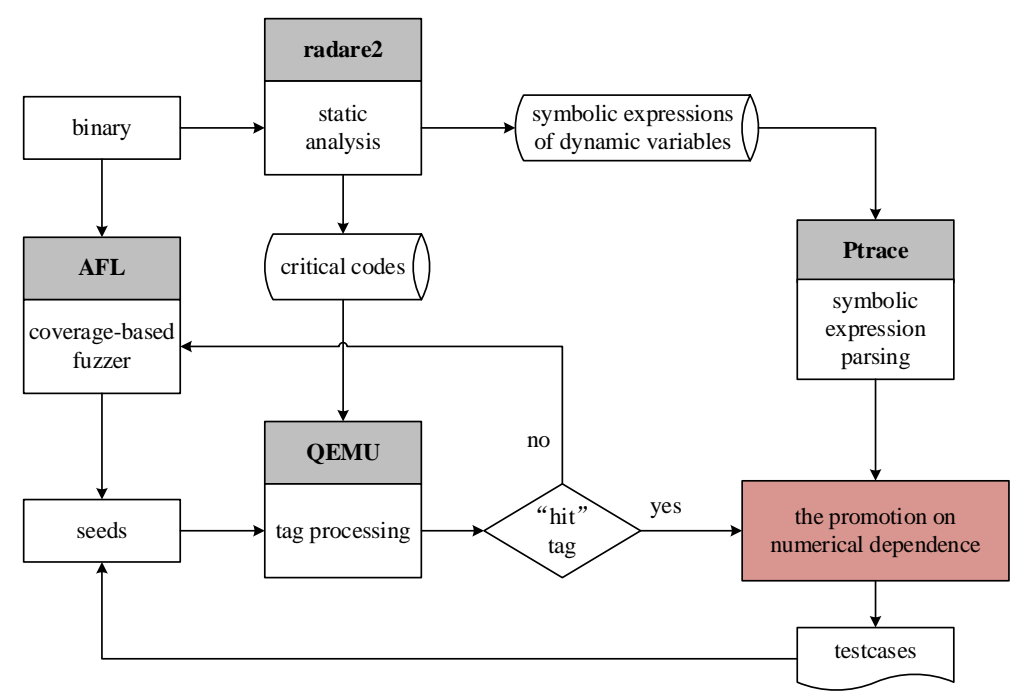

Fig. 3: The framework of ADA.

\section{Evaluation}

\subsection{Experimental Settings}

We evaluated ADA on six typical CTF benchmarks, which respectively came from the 2018 Huanghebei competition [13] and the 2019 BCTF competition [14]. To verify the effectiveness of ADA, we compared ADA with other three state-of-the-art coverage-based fuzzers, AFL, AFLFast [15] and Fairfuzz [16]. Each experiment was run for 1 hour and repeated for 5 times, and we used the average results to eliminate the randomness. It was emphasized that we did not use advanced seeds and dictionaries during the test.

All our experiments were conducted on a server with 2 Intel Xeon CPU E5-2620@ 2.10GH (24 cores in total) and 64GB memory, running 64-bit Ubuntu 16.04 LTS system.

\subsection{Evaluation Metrics}

Our experiments use two metrics to evaluate the effectiveness of the augmented fuzzing approach with a promotion on the numerical dependence. The first metric is the ability to find bugs, that is whether the fuzzer can successfully detect the bug in the program or not, and this metric is often used to estimate the performance of fuzzing. The second metric is the degree to which the numerical dependence is pushed, denoted as $\boldsymbol{p}$. The fundamental idea of ADA is to promote the dynamic variable on the execution trace to satisfy a feasible solution, thus $\boldsymbol{p}$ can rightly reflect the effect of our approach.

In this paper, we adopt the Euclidean distance [17] to quantify the second metric $\boldsymbol{p}$. For the dynamic variable's value $D$, and the feasible solution $V, \boldsymbol{p}$ is defined as $\boldsymbol{p}=1-|V-D| / D_{\max }$, where $D_{\max }$ represents the maximal Euclidean distances [17] between all possible values of the dynamic variable and the feasible solution $V$. Obviously, $\boldsymbol{p}=0$ means that the promotion module of ADA doesn't work. Inversely, $\boldsymbol{p}=$ $100 \%$ indicates that ADA finds out a testcase that can break through the comparison or satisfy the numerical dependence of triggering the bug, thereby improving the code coverage or discovering the bug.

\subsection{Results and Analysis}

For binaries listed in Table.2, the first four of them are from the 2018 Huanghebei competition [13] and the last two binaries come from the 2019 BCTF competition [14]. Specially, there are four types of numerical dependences in these binaries, including CRC encoding, format string, specific string comparison and integer overflow. Note that the numerical dependences of format string and integer overflow are directly related to bug detection, while the other two are directly associated with the code coverage.

Bug Detection Table.2 shows the results of bug detection on six programs for four fuzzers. It can be noted that coverage-guided fuzzers, AFL, AFLFast, and Fairfuzz miss all the bugs in the programs, while ADA satisfactorily uncovers them. In fact, the CTF programs are small and we find that four fuzzers have all explored the vulnerable codes in pwn02, pwn06 and A0004, which means condition $i$ has been met. 
Table. 2: Results of bug detection on six programs.

\begin{tabular}{|c|c|c|c|c|c|}
\hline \multirow{2}{*}{ Binaries } & \multirow{2}{*}{ Numerical Dependence Types } & \multicolumn{4}{|c|}{ Results of Bug Detection } \\
\cline { 3 - 6 } & & AFL & AFLFast & Fairfuzz & ADA \\
\hline pwn01 & CRC encoding & $\times$ & $\times$ & $\times$ & $\sqrt{ }$ \\
\hline pwn02 & format string & $\times$ & $\times$ & $\times$ & $\sqrt{ }$ \\
\hline pwn05 & specific string comparison & $\times$ & $\times$ & $\times$ & $\sqrt{ }$ \\
\hline pwn06 & format string & $\times$ & $\times$ & $\times$ & $\sqrt{ }$ \\
\hline A0004 & integer overflow & $\times$ & $\times$ & $\times$ & $\sqrt{ }$ \\
\hline A0008 & specific string comparison & $\times$ & $\times$ & $\times$ & $\sqrt{ }$ \\
\hline
\end{tabular}

For classic coverage-guided fuzzing, it moves on after exploring the critical codes, without adequately testing them. What's more, its random mutation algorithm makes it difficult to search for a testcase that can break through the comparison or trigger the bug. On the contrary, when the critical codes are executed, ADA will immediately add a "hit" tag to the current testcase, and perform the promotion approach on it. Considering pwn02, pwn06 and A0004, the promotion module can drive the generation of the testcases that satisfy the numerical dependence of triggering the bug, making condition ii met and uncovering the bug. For pwn01, pwn05 and A0008, the promotion module can drive the generation of the testcases that meet the numerical dependences of the CRC encoding and the string comparison, discovering the bug by improving the code coverage. Based on the above analysis, we can conclude that ADA improves the ability of bug detection on the explored paths.

Promotion Degree Fig.4 shows the degree to which the numerical dependence is pushed during the test, and the second metric $\boldsymbol{p}$ is calculated every 10 minutes. We observe that ADA enables $\boldsymbol{p}$ to reach $100 \%$ in six programs, while other fuzzers can't achieve it. Compared with ADA, we find AFL, AFLFast and Fairfuzz lose their advantages.
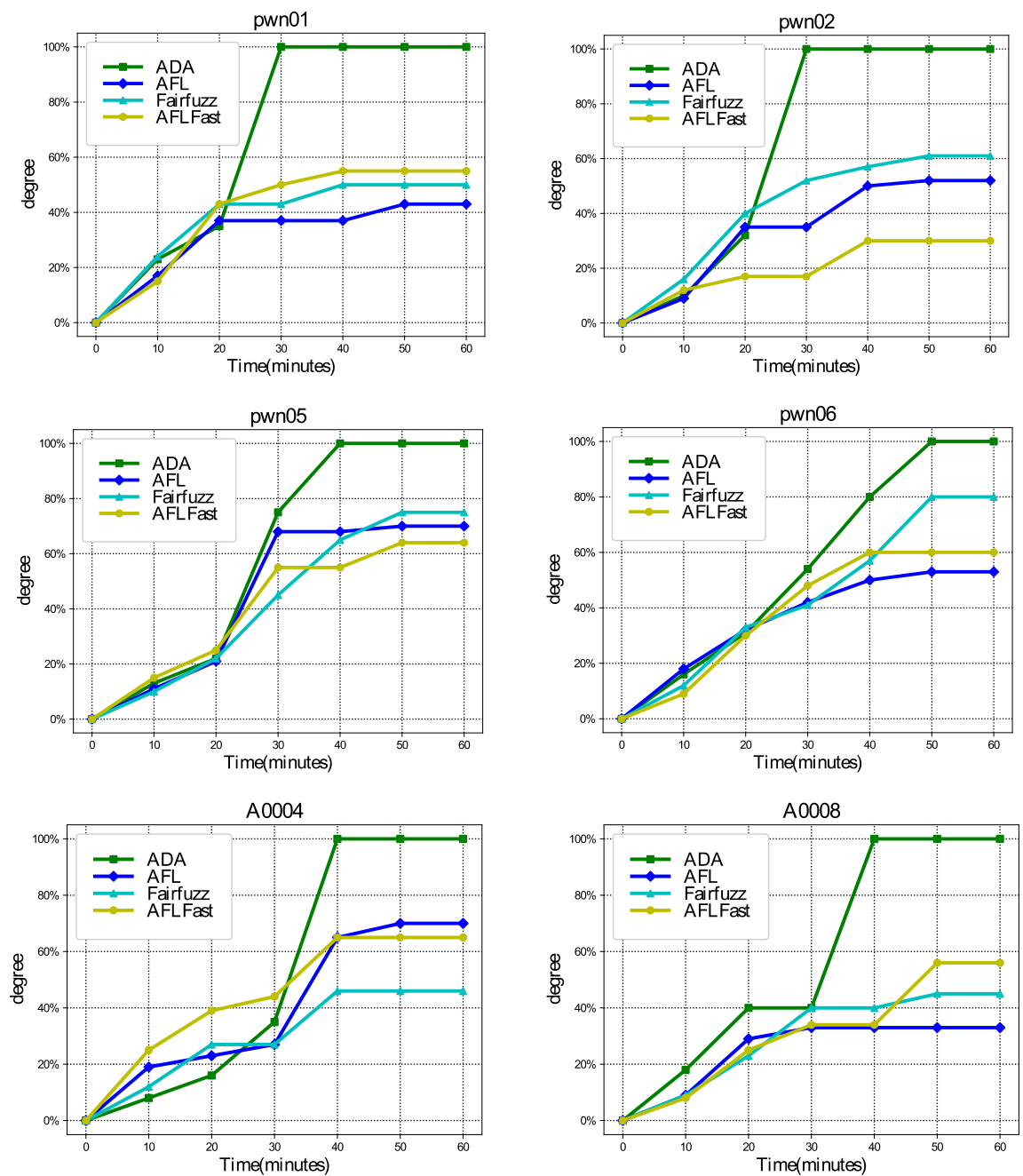

Fig. 4: The degree to which the numerical dependence is pushed during the test. 
During the test, AFL, AFLFast and Fairfuzz always mutate the input randomly, so it's hard for them to promote the numerical dependence, and the promotion degree completely depends on the ability of random testing. Contrastingly, once exploring the critical codes, ADA will allow an intended number of executions on them by launching the promotion module, which adopts a novel approximation search algorithm to generate new testcases. By introducing new guidance information, the heuristic searching process becomes less blind and it can successfully produce the testcases that meet the numerical dependence of triggering the bug or breaking through the comparison, thus ADA performs better than other fuzzers on the benchmarks.

In summary, the experimental results demonstrate that ADA outperforms AFL, AFLFast and Fairfuzz, in discovering the bugs on the executed paths. Our augmented fuzzing approach with a promotion on the numerical dependence can improve the bug detection efficiency of classic coverage-based fuzzing.

\section{Conclusion and Future Work}

Coverage-guided fuzzing is one of the most effective techniques in discovering software bugs. However, its code coverage-centric design is insufficient in bug detection. On the one hand, it quickly moves on after exploring a new path without examining the hidden defects inside. On the other hand, it always mutates the input at random, making it hard to generate a target testcase. These two limitations result in a phenomenon that some subtle bugs cannot be detected despite the vulnerable codes have been executed. To address the above problem, we propose an augmented fuzzing approach with a promotion on the numerical dependence, implemented as ADA. Unlike existing fuzzers, once exploring the critical codes, ADA will conduct ample tests on them by launching the promotion module, which utilizes a novel approximation search algorithm to produce the testcases that can satisfy the numerical dependence of triggering the bug or breaking through the comparison. What's more, we leverage a critical field identification method to assist the searching process, which can reduce the searching space of the input and help the engine to quickly produce the testcases satisfying the numerical dependence. Finally, we evaluate the performance of ADA on some benchmarks. The experimental results indicate that ADA performs better than some other state-of-the-art coverage-guided fuzzers in discovering the bugs on the executed paths, which proves the effectiveness of our approach. Furthermore, the introduced overhead by our approach is acceptable.

There are also shortcomings. Currently, our approach only supports 64-bit programs, we plan to migrate it to 32-bit programs in the future. Besides, our tool is primely designed for automated vulnerability discovery competitions, so we just evaluate the effectiveness of our approach on some CTF benchmarks, without taking the CGC programs and real software into consideration. In the follow-up work, by addressing some challenges, our approach will be extended to the condition of the CGC programs, even to real software.

\section{Acknowledgements}

We are grateful to the anonymous reviewers for their hard work, insightful comments and suggestions.

\section{References}

[1] B. P. Miller, L. Fredriksen, and B. So, “An empirical study of the reliability of UNIX utilities," Commun. ACM, vol. 33, no. 12, pp. 32-44, Dec. 1990.

[2] “OSS-Fuzz.” [Online]. Available: https://github.com/google/oss-fuzz.

[3] “American fuzzy lop.” [Online]. Available: http://lcamtuf.coredump.cx/afl/.

[4] “LibFuzzer.” [Online]. Available: https://github.com/Dor1s/libfuzzer-workshop.

[5] "Honggfuzz." [Online]. Available: http://honggfuzz.com.

[6] I. Haller, A. Slowinska, M. Neugschwandtner, and H. Bos, "Dowser: a guided fuzzer for finding buffer overflow vulnerabilities,”; login Mag. USENIX SAGE, vol. 38, no. 6, pp. 16-19, 2013.

[7] P. Godefroid, A. Kiezun, and M. Y. Levin, “Grammar-based whitebox fuzzing,” ACM SIGPLAN Not., vol. 43, no. 6, pp. 206-215, 2008.

[8] Y. Chen et al., "SAVIOR: Towards Bug-Driven Hybrid Testing,” 41th IEEE Secur. Priv. Conf., Jun. 2019.

[9] “CTF-WiKi.” [Online]. Available: https://github.com/ctf-wiki/ctf-wiki. 
[10] “Ptrace.” [Online]. Available: https://en.wikipedia.org/w/index.php?title=Ptrace\&oldid=902092021.

[11] “Radare2.” [Online]. Available: https://github.com/radare/radare2.

[12] F. Bellard, “QEMU, a Fast and Portable Dynamic Translator,” in USENIX Annual Technical Conference, 2005, pp. 41-46.

[13] “Huanghebei.” [Online]. Available: http://www.integritytech.com.cn/html/News/News_312_1.html.

[14] “BCTF2019.” [Online]. Available: https://bbs.ichunqiu.com/thread-49547-1-1.html.

[15] M. Bohme, V.-T. Pham, and A. Roychoudhury, “Coverage-Based Greybox Fuzzing as Markov Chain,” IEEE Trans. Softw. Eng., vol. 45, no. 5, pp. 489-506, May 2019.

[16] C. Lemieux and K. Sen, "FairFuzz: a targeted mutation strategy for increasing greybox fuzz testing coverage," in Proceedings of the 33rd ACM/IEEE International Conference on Automated Software Engineering - ASE 2018, 2018, pp. 475-485.

[17] “Euclidean.” [Online]. Available: https://en.wikipedia.org/w/index.php?title=Euclidean\&oldid=886192999. 